\title{
Personality traits and physical functioning: a cross-sectional multimethod facet-level analysis
}

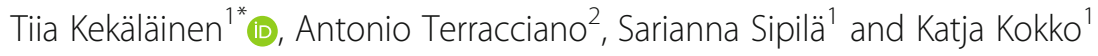

\begin{abstract}
Background: This study aimed to investigate whether personality traits and their facets are associated with a multimethods assessment of physical activity and walking performance and whether they explain the discrepancy between self-reported and accelerometer-assessed physical activity.

Methods: The participants were community-dwelling, 70-85-year-old men and women from Finland $(n=239)$ who were part of a clinical trial. Personality traits and their facets were measured using the 240-item NEO Personality Inventory-3. Physical activity was assessed using questions about frequency, intensity and duration of exercise (selfreported metabolic equivalent minutes (MET)) and by tri-axial accelerometers (light and moderate-to-vigorous physical activity and total MET-minutes). Walking performance was measured by 6-min walking distance and 10-m walking speed. Linear regression analyses were controlled for age, sex, education, body mass index, disease burden, and intervention group.
\end{abstract}

Results: The activity facet of extraversion was positively associated with self-reported MET-minutes, accelerometerassessed light physical activity and walking performance. The positive emotions facet of extraversion was positively associated with self-reported MET-minutes and walking performance. Openness and its facets and the excitement seeking facet of extraversion were positively associated with walking performance. Conscientiousness and most of its facets were associated with both physical activity and walking performance, but these associations were not statistically significant after accounting for all control variables. The impulsiveness facet of neuroticism was negatively associated with accelerometer-assessed light physical activity and walking performance, but the associations with walking performance attenuated after accounting for all control variables. Accelerometer-assessed moderate-tovigorous physical activity was not associated with personality traits or facets. Discrepancy analyses suggest that openness and the excitement-seeking facet of extraversion were associated with higher self-reported than accelerometer-assessed physical activity.

Conclusions: Consistently across methods, older adults who scored higher on facets of extraversion and conscientiousness tended to be more active and outperformed peers on walking performance. Older adults who scored higher in the facets of openness and the excitement-seeking facet of extraversion had better walking performance but also overestimated their self-reported physical activity compared to the accelerometers.

Keywords: Physical activity, Accelerometer, Walking speed, Mobility

\footnotetext{
* Correspondence: tiia.m.kekalainen@jyu.fi

${ }^{1}$ Gerontology Research Center and Faculty of Sport and Health Sciences, University of Jyväskylä, Jyväskylä, Finland

Full list of author information is available at the end of the article
}

(c) The Author(s). 2020 Open Access This article is licensed under a Creative Commons Attribution 4.0 International License, which permits use, sharing, adaptation, distribution and reproduction in any medium or format, as long as you give appropriate credit to the original author(s) and the source, provide a link to the Creative Commons licence, and indicate if changes were made. The images or other third party material in this article are included in the article's Creative Commons licence, unless indicated otherwise in a credit line to the material. If material is not included in the article's Creative Commons licence and your intended use is not permitted by statutory regulation or exceeds the permitted use, you will need to obtain permission directly from the copyright holder. To view a copy of this licence, visit http://creativecommons.org/licenses/by/4.0/ The Creative Commons Public Domain Dedication waiver (http://creativecommons.org/publicdomain/zero/1.0/) applies to the data made available in this article, unless otherwise stated in a credit line to the data. 


\section{Background}

Physical activity has many health benefits throughout the lifespan, but especially in old age it provides protection against common diseases and frailty [1]. Nevertheless, only a minority of older adults meet the recommended level of physical activity and activity tends to decrease with age [2]. Walking is the most common physical activity among older adults [3] and decline in walking speed predicts a decline in physical activity levels [4]. The large variability in physical activity levels is in part a reflection of individual differences in personality, defined as the typical pattern of feeling, thinking and behaving that characterize a person [5]. Using the Five Factor Model of personality (also known as Big Five; Table 1), several studies have found that people who score higher in extraversion, conscientiousness and openness as well as lower in neuroticism report more physical activity [7-9], have a higher walking speed [10-13], and greater aerobic capacity [13]. The information about personality correlates of physical activity and walking performance helps to identify potential risk groups who need additional attention in promotion of physical functioning. Individual differences in personality traits are known to be relatively stable throughout the adult lifespan $[14,15]$ and in general, associations between personality traits and physical activity seem to be robust between different age groups [8, 16, 17]. While current evidence suggests robust associations between personality traits and self-reported physical activity and walking speed, few studies have assessed personality traits at the level of facets (i.e., narrowly defined traits that compose each of the five broad traits; Table 1), and even fewer studies in the personality research have assessed physical activity using accelerometers. To our knowledge, no study has integrated this fragmented literature by examining the associations between personality facets and multiple measures of physical activity and walking performance in one sample.

As Table 1 illustrate, each personality factor is composed by related but distinct facets [6], and analyses at the more granular facet level can provide a more precise and deeper understanding of the associations between personality and outcomes [18-21]. An investigation at the facet-level can identify which specific facet is responsible for an association observed with the broader trait. It is possible that all facets of a trait have similar associations with an outcome, or the associations are driven by just one or a few facets, or there could be contrasting effects among facets of the same trait that could be masked by null associations at the trait level. Facet-level research can potentially improve prediction models and inform on the specific mechanisms linking psychological traits to physical activity. Research to date at the facet level suggests that the most robust positive associations are found between the activity facet of extraversion and physical activity [16, 22-28], which may even entirely explain the relationship between extraversion and physical activity [25]. The activity facet is also associated with walking speed [13]. This is not surprising because people who score high in activity tend to be busy, have a lot of energy and live at a rapid tempo [6]. The evidence for the other facets is more mixed, but, for example, the self-consciousness facet of neuroticism has been associated with lower levels of physical activity [23, $28,29]$, the self-discipline facet of conscientiousness with higher physical activity [23, 24, 29] and the dutifulness facet of conscientiousness with higher physical activity [23] and better walking performance [13]. Most of the previous studies with self-reported physical activity have analyzed only some of the facets $[24,28,29]$ and focused on younger adults [16, 24-27].

Most of the research about personality and physical activity is based on self-reported physical activity and the use of multiple measures of physical activity is necessary especially when studying older adults. Among older adults, most of the daily activity comes from light intensity activities that are easily under-reported in questionnaires [30] and, therefore, use of accelerometers can offer new information about associations between personality traits and physical activity among older adults. Accelerometers record body acceleration which is further transformed into other units, such as average daily time spend in different intensities of physical activity [31]. They can offer detailed information about daily physical activity and also overcome some common disadvantages of self-reports, such as recall bias [31]. Three previous studies on personality traits and accelerometerassessed physical activity among older adults found mixed results: Artese and colleagues [23] found that extraversion, conscientiousness and agreeableness were positively and neuroticism negatively associated with physical activity outcomes, whereas Čukić and colleagues [32] with five personality traits and our own study with extraversion and neuroticism [33] found no associations between personality traits and accelerometer-based physical activity outcomes. However, among older adults, accelerometers may underestimate the amount of moderate-to-vigorous physical activity because of lower walking speed [34]. Therefore, it would be important to study walking performance together with different measures of physical activity.

The correlation between self-reports and accelerometers vary between studies but is usually low or at most moderate [35]. Even though the discrepancy between 
Table 1 The Five Factor Model personality traits and their facets according to NEO-PI-3 [6]

\begin{tabular}{|c|c|c|c|c|}
\hline Neuroticism & Extraversion & Openness & Agreeableness & Conscientiousness \\
\hline N1 Anxiety & E1 Warmth & O1 Fantasy & A1 Trust & C1 Competence \\
\hline N2 Angry Hostility & E2 Gregariousness & O2 Aesthetics & A2 Straightforwardness & C2 Order \\
\hline N3 Depression & E3 Assertiveness & O3 Feelings & A3 Altruism & C3 Dutifulness \\
\hline N4 Self-Consciousness & E4 Activity & O4 Actions & A4 Compliance & C4 Achievement-Striving \\
\hline N5 Impulsiveness & E5 Excitement Seeking & O5 Ideas & A5 Modesty & C5 Self-Discipline \\
\hline N6 Vulnerability & E6 Positive Emotions & O6 Values & A6 Tender-Mindedness & C6 Deliberation \\
\hline
\end{tabular}

self-reported and accelerometer-assessed physical activity is well-known, individual characteristics that might explain this discrepancy are less studied. Current evidence suggests that age, sex, education and BMI are related to the discrepancy [36-38]. To our knowledge, our own previous study is thus far the only one to investigate personality and the discrepancies between physical activity measurements. Our results showed that older adults higher in neuroticism were more likely to underreport their physical activity compared to the accelerometer data [33].

The purpose of this study was to investigate whether personality traits and their facets 1 ) are associated with accelerometer-assessed and self-reported physical activity and walking performance, and 2) explain the discrepancy between accelerometer-assessed and self-reported physical activity. We expected that extraversion, openness and conscientiousness have positive associations and neuroticism a negative association with physical functioning. In the facet level, the strongest associations was expected to found between the activity facet of extraversion and physical functioning. For other facets the approach was exploratory, because to our knowledge, there is only one previous study about associations of all facet-level personality traits with walking performance [13] and one with accelerometer-assessed physical activity [23]. By addressing these questions, we aim to integrate evidence from multiple methods to advance knowledge on the role of personality traits in older adults' physical functioning.

\section{Methods}

\section{Participants}

This study examined cross-sectional data collected in a post-intervention assessment of a randomized controlled trial "Promoting safe walking among older people: the effects of a physical and cognitive training intervention vs. physical training alone on mobility and falls among older community-dwelling men and women" (the PASS WORD study) [39]. The study was approved by the Ethics committee of the Central Finland Health Care District and all participants provided their written informed consent for participation. The study has been registered in the International Standard Randomized
Controlled Trial Number Register: http/www.isrctn. com/ISRCTN52388040.

The participants were randomly selected from the Finnish National Registry. Recruitment started with an information letter sent to a random sample of 70- to 85year-old community-dwelling older adults living in the city of Jyväskylä, Finland, followed up by a phone call. During the phone interview, willingness to participate in the study was confirmed and participants were screened for inclusion and exclusion criteria. The inclusion criteria at baseline were being at most moderately active (walking $<150 \mathrm{~min} /$ week, no regular resistance training), able to walk $500 \mathrm{~m}$ without assistance and to score $\geq 24$ points on the Mini Mental State Examination. Exclusion criteria included severe chronic condition, medication, or other factors that may affect study participation, excessive alcohol use, difficulties in communication due to severe hearing or vision problems and another family member participating in the study [39]. The sample size was based on a pre-trial power analysis for primary outcomes of the trial (walking speed and falls rate) [39]. Participants were randomized to two groups, a combined physical and cognitive training vs physical training alone. As such, both groups participated in the same 12month physical training intervention and both groups engaged in levels of physical training that matched the recommended guidelines including aerobic training (mostly walking), progressive resistance and balance training [39].

We have previously reported the baseline associations of extraversion and neuroticism (measured using a modified version of the Eysenck's short Personality Inventory) with physical activity in the same sample [33]. This study used data only from post-intervention measurements because the NEO-Personality Inventory-3 (NEO-PI-3) was administered only in the postintervention. The analyses were restricted to those participants $(n=239)$ who completed the NEO-PI-3. All of these participants had participated in physical training alone or combined physical and cognitive training during the last 12 months. Compared to the rest of the original sample $(n=75)$, the participants included in this study $(n=239)$ were younger (mean age $74.20 \pm 3.71$ vs. $75.33 \pm 4.05, p=.021)$ and had a higher level of 
education ( $88 \%$ vs. $73 \%$ in the medium or high education group, $p=.007)$.

\section{Measurements}

The NEO-PI-3 was used to assess personality traits and facets $[6,40]$. It has 240 items, 48 for each personality trait and 8 for each facet (see Table 1). The response scale is from $0=$ strongly disagree to $4=$ strongly agree. Participants were given a paper version of the NEO-PI-3 in the last training session and asked to return it when they came for the post-intervention measurements. Of 289 participants, 244 participants returned the NEO-PI3 questionnaire. Three questionnaires were empty, and two participants had more than 40 missing values (the exclusion limit in the NEO-PI-3 manual [40]), so those participants $(n=5)$ were excluded from this study. Of those 239 participants who had filled the NEO-PI-3 questionnaire at least partly, 193 (81\%) had filled it completely without any missing values and the rest of the participants $(n=46)$ had seven missing values at the most. These were imputed with the neutral option 2 [40]. Sum scores for each facet and each trait were calculated and these raw scores were used in the analyses. Raw scores were transformed to T-scores using combined sex-norms reported in the NEO-PI-3 Finnish version manual [40] for comparison purposes. The Cronbach's alphas were 0.87 for neuroticism, 0.80 for extraversion, 0.75 for openness, 0.76 for agreeableness and 0.85 for conscientiousness.

Physical activity was assessed by both accelerometers and self-reports. Participants were asked to wear a triaxial accelerometer, model UKK RM42 (UKK, Tampere, Finland), in an elastic band on their right hip during waking hours for seven consecutive days except during water-based activities [41]. The accelerometer stores acceleration at $100 \mathrm{~Hz}$ sampling rate with 13-bit A/D conversion of the $\pm 16 \mathrm{~g}$ range. The raw acceleration data were analyzed with a custom-written MATLAB (version R2016b, The MathWorks Inc., Natick MA, USA) script for mean amplitude deviation (MAD) with a previously published algorithm [42]. Mean daily minutes for sedentary, light, moderate and vigorous intensity activity were calculated with previously validated cut-off points $[42,43]$. Acceptable accelerometer data (at least 3 days with at least a 10-h wearing time) were available for 266 participants (wearing time mean $13.9 \mathrm{~h} / \mathrm{d} \pm 1.3,90 \%$ of participants with 6 or 7 valid days). In the present study, information about light, moderate and vigorous activity was used. Because the amount of vigorous intensity activity was almost non-existent (mean $0.11 \mathrm{~min} / \mathrm{d} \pm$ 0.57 ), it was merged with moderate activity (MVPA). Three questions asking monthly frequency, duration and intensity of exercise were used to calculate daily selfreported MET-minutes [44].
For discrepancy analysis, average daily metabolic equivalent (MET)-minutes from accelerometer data were calculated with the common thresholds for light, moderate and vigorous activity: $1.6^{*}$ light +3 moderate +6 *vigorous physical activity [45]. Because MET-minutes from accelerometer (all daily activity) and self-reports (questions about exercise) were not directly comparable, standardized values were used. Standardized MET-minutes from accelerometer data were subtracted from standardized self-reported MET-minutes and such difference was used as the outcome in the analyses. The positive discrepancy score indicates that a participant had reported higher MET-minutes than what was assessed by the accelerometers compared to the other participants.

Two walking performance tests were used in this study: the 6-min walking test evaluates community walking [46] and the 10-m walking test evaluates maximum walking speed. In the 6-min walking test, participants walked up and down a 20-m circuit without resting. They were encouraged to walk as far as possible for 6 min [46]. In the 10-m walking test, participants were asked to walk as fast as possible over the 10-m course [39]. The time was measured by photocells and the maximum walking speed $(\mathrm{m} / \mathrm{s})$ was calculated from the best performance of two attempts.

The background variables included sex, age, body mass index (BMI), education and chronic diseases. Sex and date of birth were drawn from the Finnish Population Registry. BMI was calculated from staff-measured height and weight. Education was categorized to low (at most primary school), medium (middle school, folk high school, vocational school or secondary school) and high (college or university degree). Self-report chronic diseases diagnosed by physicians were reported at baseline and were updated with new verified diagnoses that occurred during the intervention. Answers to questions on chronic diseases were categorized into metabolic, cardiovascular, pulmonary, musculoskeletal, neurological and mental diseases. Number of disease categories was calculated and used in analyses with a categorization of $0,1,2$ and 3 or more disease categories.

\section{Statistical analyses}

All statistical analyses were carried out using SPSS, version 25 (IBM Corp., Armonk, NY). Demographic characteristics of the study sample were described using proportions, means and standard deviations and minimum and maximum values.

Associations of personality traits and their facets with physical activity and walking speed were analyzed by linear regression. Every trait and facet were analyzed in separate models to avoid multicollinearity. The models with all five traits in the same model are presented in supplementary material (see Additional file 1, Table S1). 
Table 2 Descriptive statistics for study variables $(n=239)$

\begin{tabular}{lll}
\hline & Mean \pm SD & Min - Max \\
\hline Accelerometer: Light physical activity min/d & $65.00-423.00$ \\
Accelerometer: MVPA min/d & $206.56 \pm 66.17$ & $0-125.14$ \\
Accelerometer: MET min/d & $32.15 \pm 21.01$ & $107.00-786.89$ \\
Self-reported MET min/d & $427.27 \pm 129.84$ & $0.47-750.00$ \\
6-min walking distance m & $115.60 \pm 108.09$ & $249-736$ \\
10-m walking speed m/s & $521.13 \pm 92.76$ & $0.96-3.22$ \\
Neuroticism & $2.08 \pm 0.42$ & $17-133$ \\
Extraversion & $73.18 \pm 21.82$ & $43-169$ \\
Openness & $95.11 \pm 20.47$ & $63-181$ \\
Agreeableness & $109.18 \pm 18.98$ & $77-170$ \\
Conscientiousness & $128.54 \pm 15.94$ & $64-166$ \\
\hline
\end{tabular}

MVPA moderato-to-vigorous physical activity

${ }^{a}$ Calculated with a formula $1.6^{*}$ light physical activity $+3^{*}$ moderate physical activity $+6^{*}$ vigorous physical activity

Sex, age, BMI, education and chronic diseases were used as covariates in line with previous studies assessing associations between personality traits and physical performance $[8,10,13,23,32]$.

Linear regression models for each physical activity and walking performance outcomes as well as to the discrepancy score were computed in two phases: the first model (M1) included a trait or facet and sex and age as covariates while the rest of the covariates (BMI, chronic diseases, education and the intervention group) were included in the second model (M2). M2 models for light physical activity also included MVPA as a covariate and M2 models for MVPA also included light physical activity as a covariate. Because the variable had a rightskewed distribution in self-reported MET-minutes, the

Table 3 Pearson bi-variate correlations between study variables

\begin{tabular}{|c|c|c|c|c|c|c|c|c|c|c|c|}
\hline & 1. & 2. & 3. & 4. & 5. & 6. & 7. & 8. & 9. & 10. & 11. \\
\hline \multicolumn{12}{|c|}{ Accelerometer- based physical activity } \\
\hline \multicolumn{12}{|l|}{ 1. Light } \\
\hline 2. MVPA & .12 & & & & & & & & & & \\
\hline 3. MET min & $.87^{*}$ & $.59^{*}$ & & & & & & & & & \\
\hline \multicolumn{12}{|c|}{ Self-reported physical activity } \\
\hline 4. MET min & $.25^{*}$ & $.40^{*}$ & $.40^{*}$ & & & & & & & & \\
\hline \multicolumn{12}{|l|}{ Walking tests } \\
\hline 5. 6-min & $.20^{*}$ & $.48^{*}$ & $.41^{*}$ & $.32^{*}$ & & & & & & & \\
\hline 6. 10-m & $.14^{*}$ & $.31^{*}$ & $.27^{*}$ & $.19^{*}$ & $.83^{*}$ & & & & & & \\
\hline \multicolumn{12}{|l|}{ Personality traits } \\
\hline 7. Neuroticism & -.03 & .02 & -.02 & -.12 & $-.21^{*}$ & $-.15^{*}$ & & & & & \\
\hline 8. Extraversion & $.16^{*}$ & -.03 & .11 & $.19^{*}$ & $.15^{*}$ & $.15^{*}$ & $-.30^{*}$ & & & & \\
\hline 9. Openness & .00 & .05 & .03 & $.13^{*}$ & $.14^{*}$ & .12 & .04 & $.42^{*}$ & & & \\
\hline 10. Agreeableness & .01 & -.05 & -.01 & .06 & -.00 & -.06 & $-.32^{*}$ & -.01 & .10 & & \\
\hline 11. Conscientiousness & $.19^{*}$ & .04 & $.18^{*}$ & $.21^{*}$ & $.24^{*}$ & $.17^{*}$ & $-.55^{*}$ & $.24^{*}$ & -.11 & $.29^{*}$ & \\
\hline \multicolumn{12}{|l|}{ Covariates } \\
\hline 12. BMI & $-.31^{*}$ & $-.14^{*}$ & $-.31^{*}$ & $-.18^{*}$ & $-.35^{*}$ & $-.19^{*}$ & .04 & .01 & -.12 & -.04 & $-.17^{*}$ \\
\hline 13. Chronic diseases & .04 & $-.20^{*}$ & -.07 & -.08 & $-.27^{*}$ & $-.16^{*}$ & $.16^{*}$ & .02 & -.06 & -.09 & -.12 \\
\hline 13. Age & -.08 & $-.28^{*}$ & $-.20^{*}$ & $-.22^{*}$ & $-.34^{*}$ & $-.30^{*}$ & .12 & -.10 & -.00 & .02 & $-.13^{*}$ \\
\hline 14. Education & .11 & .07 & .12 & -.00 & $.13^{*}$ & $.16^{*}$ & .04 & .08 & $.28^{*}$ & -.08 & -.06 \\
\hline
\end{tabular}

MVPA moderate-to-vigorous physical activity, $M E T$ metabolic equivalent, $B M I$ body mass index ${ }^{*} p<.05$ 
Table 4 Associations of personality traits and facets with walking performance, physical activity and discrepancy between physical activity measurements

\begin{tabular}{|c|c|c|c|c|c|c|c|c|c|c|}
\hline & \multicolumn{2}{|c|}{$\begin{array}{l}\text { Walking distance 6- } \\
\text { min }\end{array}$} & \multicolumn{2}{|c|}{$\begin{array}{l}\text { Walking speed } 10- \\
\mathrm{m}\end{array}$} & \multicolumn{2}{|c|}{$\begin{array}{l}\text { Self-reported physical } \\
\text { activity }\end{array}$} & \multicolumn{2}{|c|}{$\begin{array}{l}\text { Light physical } \\
\text { activity }\end{array}$} & \multicolumn{2}{|c|}{ Discrepancy } \\
\hline & M1 & M2 & M1 & M2 & M1 & M2 & M1 & M2 & M1 & M2 \\
\hline Neuroticism & $-.13^{*}$ & -.11 & -.05 & -.02 & -.09 & -.08 & -.05 & -.06 & -.05 & -.05 \\
\hline Extraversion & $.14^{*}$ & $.13^{*}$ & $.15^{*}$ & $.13^{*}$ & $.17^{* *}$ & $.18^{* *}$ & $.15^{*}$ & $.14^{*}$ & .08 & .09 \\
\hline Openness & $.25^{* * *}$ & $.18^{*}$ & $.27^{* * *}$ & $.21^{* * *}$ & $.15^{*}$ & $.14^{*}$ & -.05 & -.12 & $.19 * *$ & $.22^{* *}$ \\
\hline Agreeableness & .07 & .06 & .04 & .04 & .08 & .06 & -.02 & -.01 & .06 & .05 \\
\hline Conscientiousness & $.17^{* *}$ & .09 & .09 & .04 & $.18^{* *}$ & $.14^{*}$ & $.20 * *$ & $.17^{*}$ & -.05 & -.03 \\
\hline N1 Anxiety & -.03 & -.03 & .04 & .04 & -.04 & -.05 & .11 & .08 & $-.18^{*}$ & $-.16^{*}$ \\
\hline N2 Angry Hostility & -.02 & -.01 & .04 & .04 & -.05 & -.05 & .05 & .02 & -.10 & -.09 \\
\hline N3 Depression & $-.15^{*}$ & -.09 & -.08 & -.03 & $-.13^{*}$ & -.12 & -.05 & -.05 & -.08 & -.08 \\
\hline N4 Self-Consciousness & -.08 & -.03 & -.04 & .00 & .01 & .01 & -.01 & .01 & -.01 & -.02 \\
\hline N5 Impulsiveness & $-.21^{* * *}$ & -.10 & $-.15^{*}$ & -.08 & -.12 & -.07 & $-.29^{* * *}$ & $-.24^{* * *}$ & $.19^{*}$ & $.18^{*}$ \\
\hline N6 Vulnerability & -.12 & -.09 & -.07 & -.05 & -.08 & -.09 & -.04 & -.06 & -.06 & -.04 \\
\hline E1 Warmth & .06 & .08 & .08 & .09 & .08 & .09 & .06 & .10 & .06 & .05 \\
\hline E2 Gregariousness & .00 & .02 & .03 & .02 & .08 & .10 & .03 & .05 & .03 & .02 \\
\hline E3 Assertiveness & .05 & .02 & .08 & .03 & .09 & .10 & .12 & .10 & .02 & .04 \\
\hline E4 Activity & $.19^{* *}$ & $.14^{*}$ & $.16^{* *}$ & .11 & $.20^{* *}$ & $.19^{* *}$ & $.28^{* * *}$ & $.24 * * *$ & -.02 & .01 \\
\hline E5 Excitement Seeking & $.12^{*}$ & .10 & $.18^{* *}$ & $.15^{* *}$ & .12 & .11 & .03 & .00 & $.17^{*}$ & $.19^{* *}$ \\
\hline E6 Positive Emotions & $.18^{* *}$ & $.21 * * *$ & $.14^{*}$ & $.16^{* *}$ & $.19^{* *}$ & $.20 * *$ & .12 & $.14^{*}$ & .07 & .07 \\
\hline O1 Fantasy & .09 & .10 & $.13^{*}$ & $.13^{*}$ & .04 & .04 & -.09 & -.10 & $.18^{* *}$ & $.19^{* *}$ \\
\hline $\mathrm{O} 2$ Aesthetics & $.18^{* *}$ & $.15^{*}$ & $.21^{* *}$ & $.17^{* *}$ & $.16^{*}$ & $.15^{*}$ & -.02 & -.09 & .12 & $.16^{* *}$ \\
\hline O3 Feelings & $.19^{* *}$ & $.18^{*}$ & $.21 * *$ & $.19 * *$ & $.16^{*}$ & $.17^{*}$ & .04 & .03 & .08 & .09 \\
\hline O4 Actions & .11 & .04 & .07 & .01 & .11 & .09 & -.03 & -.08 & .12 & $.14^{*}$ \\
\hline O5 Ideas & $.19^{* *}$ & $.12^{*}$ & $.23 * * *$ & $.16^{*}$ & .07 & .07 & -.07 & -.11 & $.16^{*}$ & $.18^{* *}$ \\
\hline O6 Values & $.20^{* *}$ & .10 & $.21 * * *$ & $.13^{*}$ & .05 & .02 & .01 & -.06 & .02 & .05 \\
\hline A1 Trust & $.17^{* *}$ & $.12^{*}$ & .10 & .07 & .11 & .09 & -.01 & -.03 & .09 & .09 \\
\hline A2 Straightforwardness & .08 & .02 & -.01 & -.04 & .08 & .05 & .05 & .02 & .00 & .01 \\
\hline A3 Altruism & .02 & .07 & .07 & .10 & -.03 & -.01 & .02 & .05 & .04 & .02 \\
\hline A4 Compliance & -.01 & .02 & -.04 & .00 & .03 & .03 & -.10 & -.06 & .07 & .05 \\
\hline A5 Modesty & -.08 & -.06 & -.07 & -.04 & -.04 & -.04 & -.04 & -.02 & -.07 & -.09 \\
\hline A6 Tender-mindedness & .08 & .06 & .11 & .08 & $.14^{*}$ & $.13^{*}$ & .02 & .01 & .11 & .12 \\
\hline C1 Competence & $.20^{* *}$ & $.13^{*}$ & $.17^{* *}$ & $.11^{*}$ & $.18^{* *}$ & $.15^{*}$ & $.17^{*}$ & $.14^{*}$ & .03 & .04 \\
\hline C2 Order & .05 & -.01 & -.04 & -.07 & $.13^{*}$ & .10 & .09 & .05 & -.04 & -.02 \\
\hline C3 Dutifulness & $.17^{* *}$ & .10 & .10 & .05 & .04 & .02 & $.15^{*}$ & $.14^{*}$ & -.07 & -.06 \\
\hline C4 Achievement-Striving & $.13^{*}$ & .06 & .09 & .05 & $.13^{*}$ & .10 & $.23 * *$ & $.18^{* *}$ & -.03 & -.00 \\
\hline C5 Self-Discipline & $.19^{* *}$ & .11 & .07 & .02 & $.21^{* *}$ & $.17^{*}$ & $.14^{*}$ & .10 & -.03 & -.01 \\
\hline C6 Deliberation & .10 & .03 & .07 & .03 & $.13^{*}$ & .10 & $.17^{* *}$ & $.15^{*}$ & -.09 & -.08 \\
\hline
\end{tabular}

Every trait and facet analyzed in the separate linear regression model; standardized beta-coefficients represented

${ }^{a}$ Standardized self-reported MET-minutes - standardized accelerometer-assessed MET-minutes. M1 = model including sex and age as covariates. M2 = model including sex, age, education, BMl, diseases and intervention group. M2 for light physical activity controlled also with moderate-to-vigorous physical activity Bolded beta-coefficients remained statistically significant after Benjamini-Hochberg procedure.

${ }^{* * *} p<.001,{ }^{* *} p<.01,{ }^{*} p<.05$ 
logarithm transformation was used in linear regression analysis.

The Benjamini-Hochberg procedure was used to correct the high number of comparisons with a false discovery rate of $0.10[47,48]$. The procedure was conducted first to models with age and sex as covariates (M1) with six outcomes (walk $6 \mathrm{~min}$, walk $10 \mathrm{~m}$, light physical activity, MVPA, self-reported MET-minutes and discrepancy score) including a total of 210 models (6 outcomes* (5 traits +30 facets) $)$ and then similarly to 210 models with all covariates (M2).

\section{Results}

The descriptive statistics for main study variables are presented in Table 2 and for the facets in supplementary material (Additional file 2, Table S2). A total of 141 (59\%) women and 98 (41\%) men with a mean age of $74.71 \pm 3.72$ and mean BMI of $27.76 \pm 4.66$ participated in this study. Most of the participants (66\%) were in the medium education group, $12 \%$ had at most a primary degree (low) and $22 \%$ had a college or university degree (high). Only $13 \%$ did not report a chronic metabolic, cardiovascular, pulmonary, musculoskeletal, neurological or mental disease; $32 \%$ reported one, $32 \%$ reported two, and $23 \%$ reported three or more chronic disease categories. Compared to a Finnish normative sample [40], our sample of older adults tended to score low on neuroticism (T-score: $42.76 \pm 7.84$ ), high on agreeableness $(58.16 \pm 7.80)$, and about average on extraversion (49.50 \pm 8.28$)$, openness $(48.58 \pm 9.00)$, and conscientiousness $(53.07 \pm 9.29)$. The correlations between main study variables are presented in Table 3 and for facets in supplementary material (Additional file 3, Table S3).

\section{Physical activity}

At the broad five trait level, extraversion was positively associated with self-reported MET-minutes and conscientiousness with both light physical activity and self-reported MET-minutes (Table 4 and Figs. 1, 2, 3, 4 and 5). The association of conscientiousness with both outcomes became statistically non-significant in the model with all control variables (M2) after Benjamini-Hochberg corrections. Neuroticism and agreeableness were not associated with any physical activity outcomes. None of the traits was associated with MVPA (Additional file 4, Table S4).

At the facet level, higher scores in most facets of conscientiousness were associated with higher physical activity (Table 4, Fig. 5): C1 competence was associated

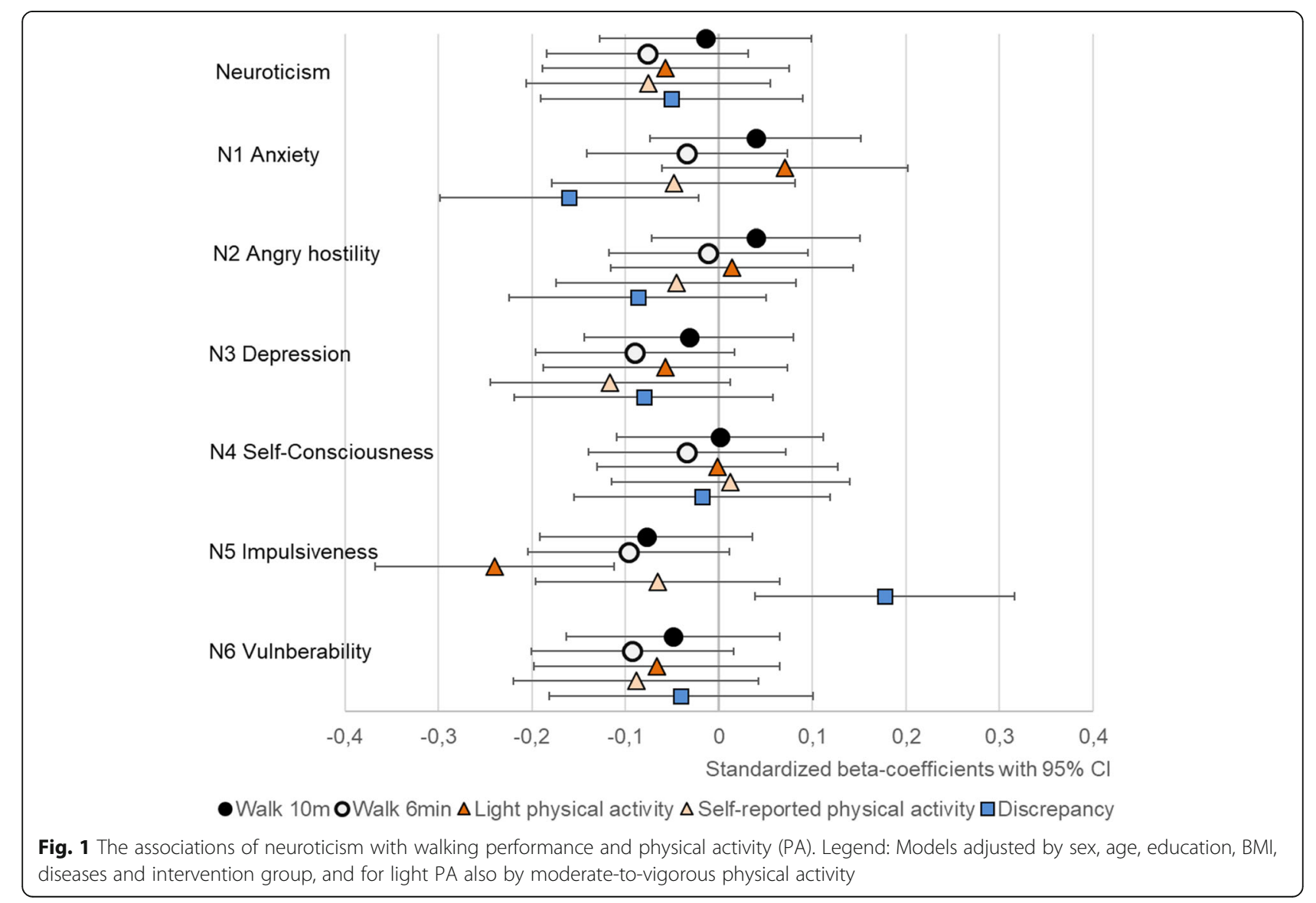




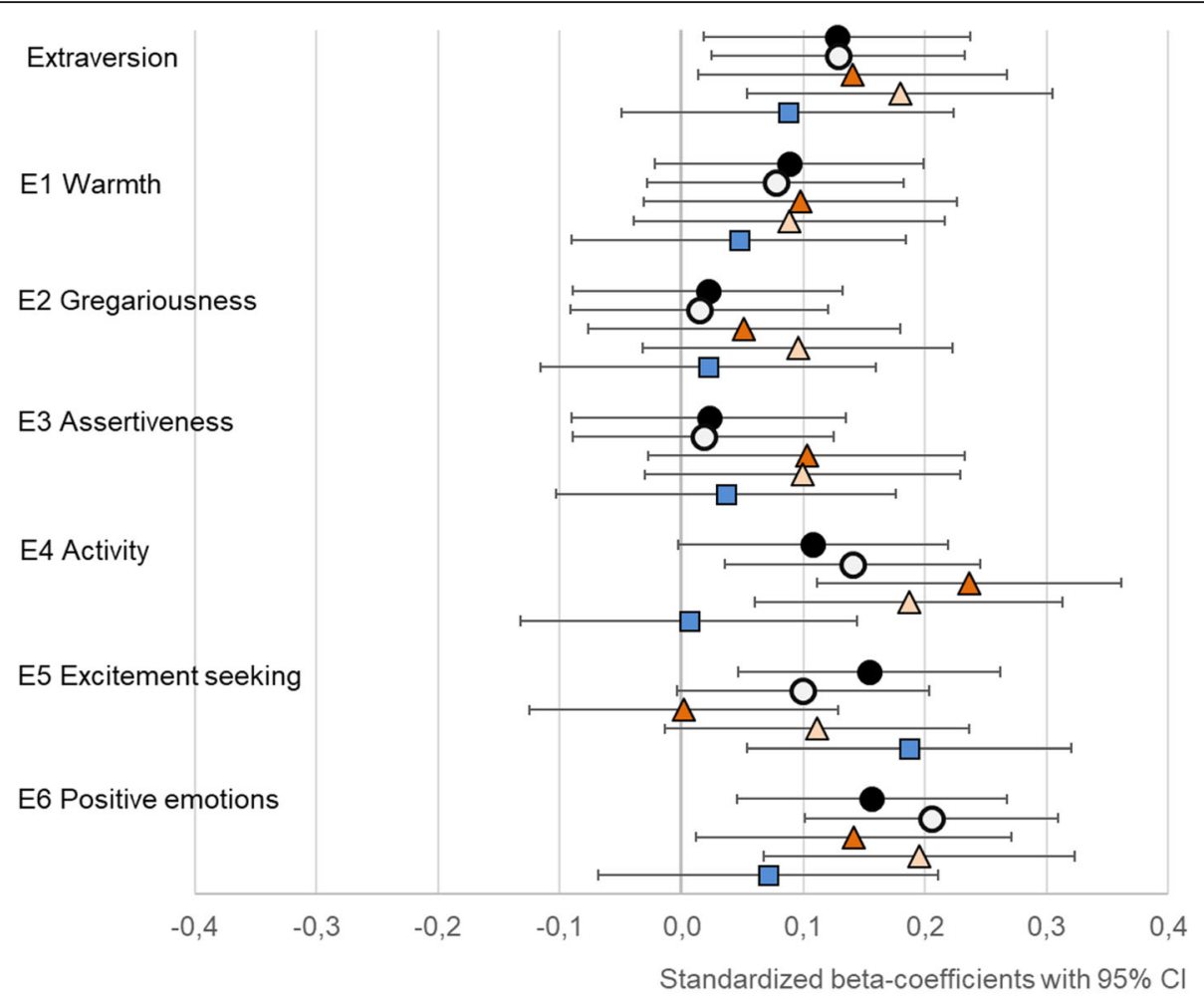

Walk $10 \mathrm{~m} \mathbf{O}$ Walk $6 \mathrm{~min} \Delta$ Light physical activity $\Delta$ Self-reported physical activity $\square$ Discrepancy

Fig. 2 The associations of extraversion with walking performance and physical activity (PA). Legend: Models adjusted by sex, age, education, BMI, diseases and intervention group, and for light PA also by moderate-to-vigorous physical activity

with both self-reported MET-minutes and light physical activity, C4 achievement-striving and C6 deliberation with light physical activity and $\mathrm{C} 5$ self-discipline with self-reported MET-minutes. The inclusion of all control variables in M2 models reduced the associations of conscientiousness facets with both self-reported METminutes and light physical activity. After the BenjaminiHochberg corrections, only C4 achievement-striving remained statistically significant predictor of light physical activity and none of the facets for self-reported MET-minutes. The E4 activity facet of extraversion was consistently associated with both light physical activity and self-reported MET-minutes and E6 positive emotions facet with self-reported MET-minutes in all models (Table 4, Fig. 2). Of other facets, N5 impulsiveness was negatively associated with light physical activity in both models. The only other facet-level association was found between $\mathrm{O} 3$ feelings and self-reported MET-minutes, but the associations became non-significant after accounting for all control variables (M2) and Benjamini-Hochberg corrections.

\section{Walking performance}

Openness and most of its facets had the strongest associations with walking performance (Table 4 and Fig. 3).
The extraversion facets E4 activity, E5 excitement seeking and E6 positive emotions had positive associations with walking performance tests, but E5 excitement seeking was associated only with walking speed and the association of E4 activity with walking speed was not statistically significant in M2 (Table 4, Fig. 2). The positive association between conscientiousness and its facets with walking distance did not remain statistically significant in M2. Similarly, the negative association between N5 impulsiveness and both walking performance tests became statistically non-significant after accounting for BMI, disease burden, education and intervention group. Of other facets, A1 trust, had positive association with walking performance in M1, but the associations became statistically non-significant in M2 after the BenjaminiHochberg procedure (Table 4).

\section{Discrepancy}

Participants who scored higher in openness (at least in O1 Fantasy and O5 Ideas) reported more physical activity than what was assessed by the accelerometers (Table 4, Fig. 3). None of the other traits was associated with discrepancy in the trait-level, but higher scores in N5 impulsiveness and E5 excitement seeking were associated with reporting more and $\mathrm{N} 1$ anxiety 


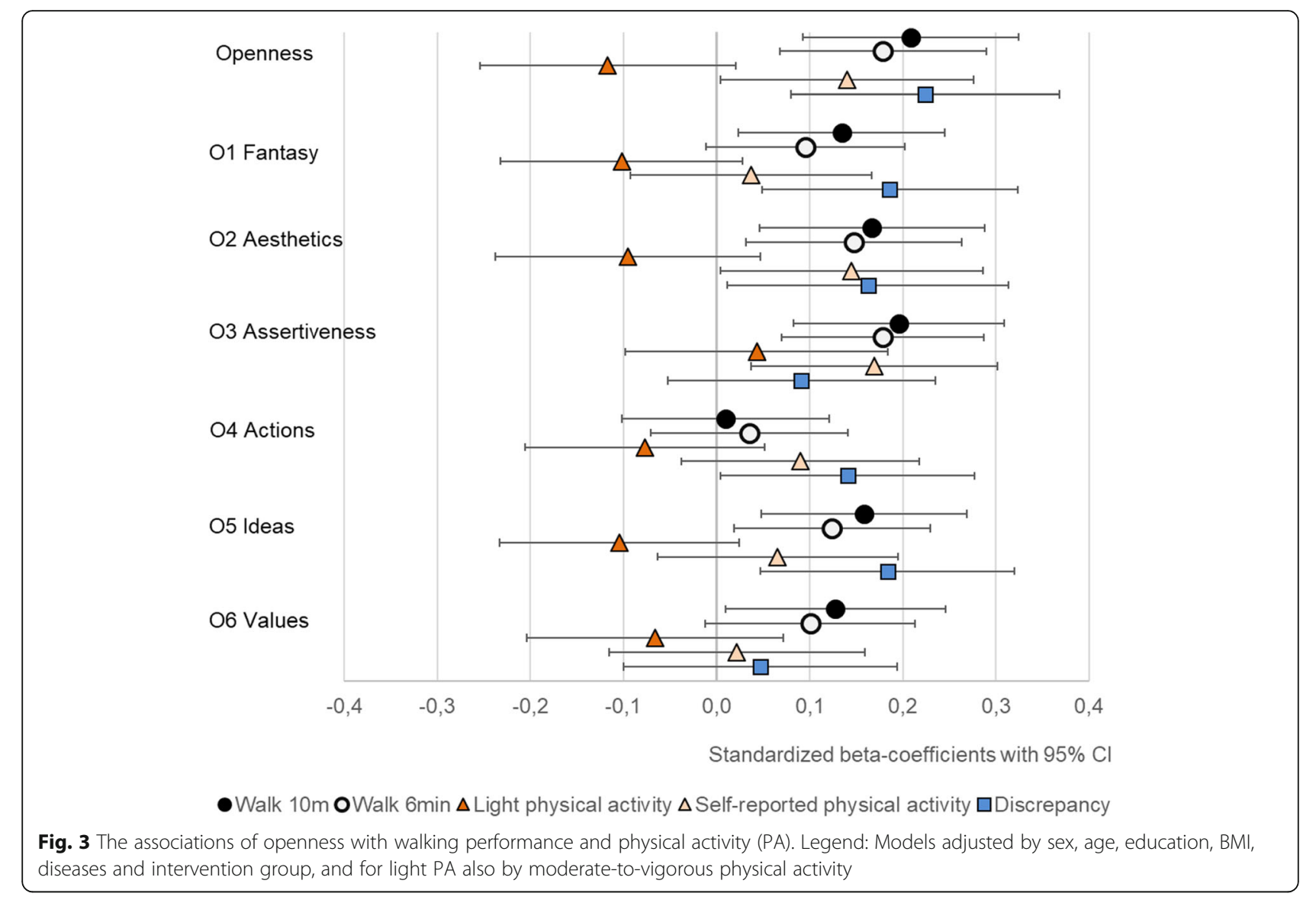

less physical activity compared to the accelerometers. The associations of N1 anxiety and N5 impulsiveness did not remain statistically significant in M2 after the Benjamini-Hochberg procedure.

\section{Discussion}

Among the significant findings, the activity and positive emotion facets of extraversion and the facets of conscientiousness were positively associated with physical activity and walking performance. These findings suggest consistent associations with physical performance regardless of the way it is assessed. There were also contrasting findings across methods, and discrepancy analyses indicate that individuals who scored higher in openness and excitement-seeking tend to overestimate their physical activity levels using self-reports compared to the accelerometers but still had better walking performance. Next, we discuss the findings for each trait.

The negative association between neuroticism and physical activity found in previous studies $[8,9]$ was weaker and non-significant in the present study. The non-significant associations may be in part due to the lower scores in neuroticism of this sample compared to the Finnish adult population [40]. However, the facetlevel analysis of neuroticism revealed some interesting findings. The impulsiveness facet had one of the strongest associations with physical activity: participants who scored higher in impulsiveness had lower accelerometerassessed light physical activity and lower walking distance but did not have significantly lower self-reported physical activity. This discrepancy indicates that more impulsive individuals may over-report their level of physical activity. Impulsiveness is related to unfavorable health behaviors, such as obesity [49], and people who score high in impulsiveness might seek immediate rewards from eating or sedentary behavior rather than delayed ones from physical activity. Our previous results with the baseline sample using a modified version of the Eysenck's short personality inventory showed that individuals who scored high in neuroticism were more likely to underreport their physical activity compared to the accelerometer data [33]. This result, which conflicts with the current findings for impulsiveness but accords with the findings for anxiety, highlights the importance of facet-level analysis.

Extraversion was consistently associated with physical activity and walking performance in line with previous studies $[8-13,23]$, but only the strongest association with self-reported physical activity remained statistically significant after the correction for multiple tests. In line 


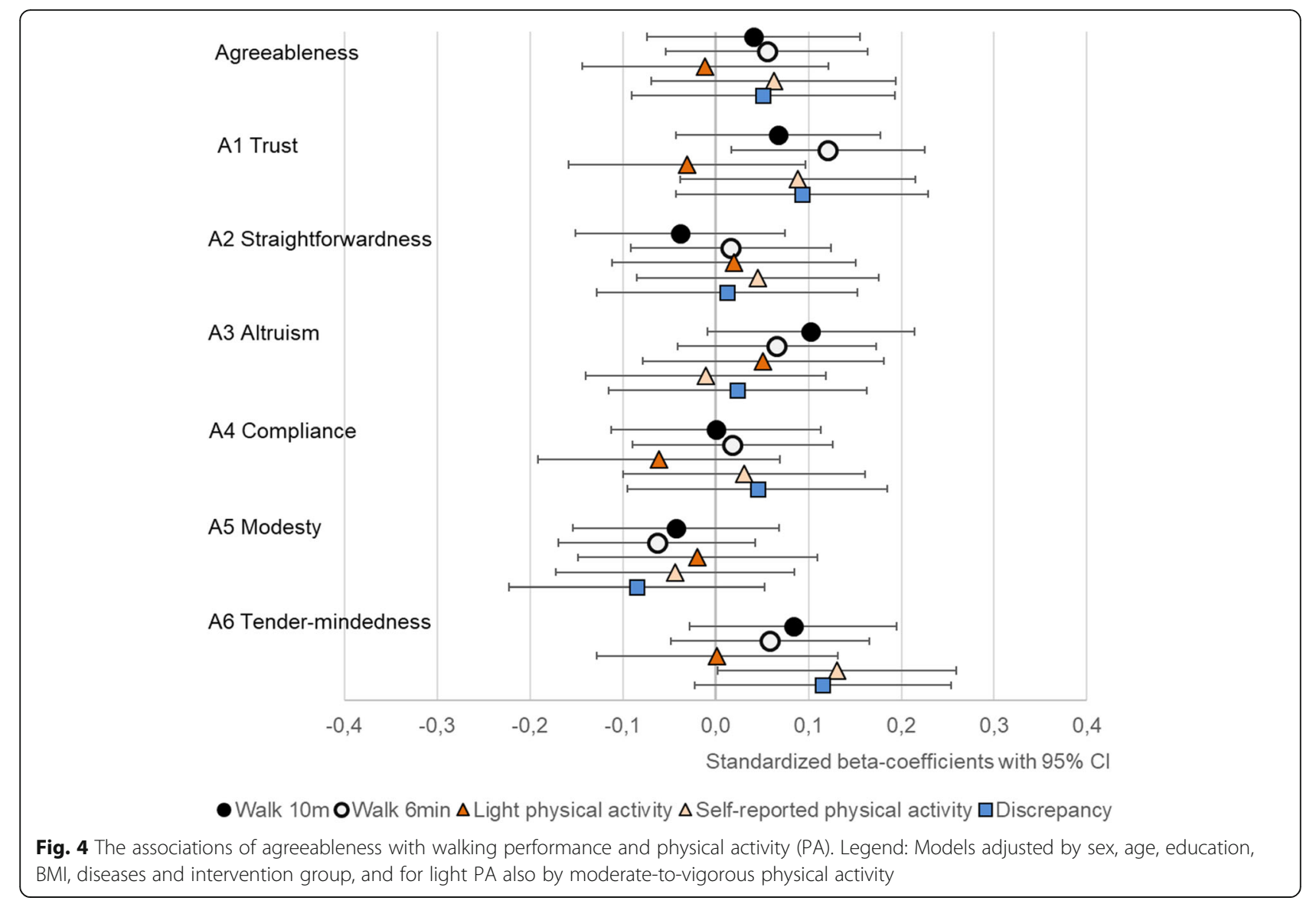

with the expectations, the findings support the importance of the activity facet in the relationship between extraversion and physical activity [23-25, 28]. In addition to activity, positive emotions and excitementseeking were linked to the outcomes: positive emotions with both self-reported physical activity and walking performance and excitement-seeking with walking performance and discrepancy. These three facets of extraversion describe the tendencies of being energetic and optimistic and seeking excitement and stimulus [6]. It seems that this side of extraversion is more important for physical performance than the tendencies related to social relationships captured by the three other facets of extraversion.

The results for openness in this study were in line with previous studies showing a weak positive association between openness and self-reported physical activity $[8,9]$ and no association with accelerometer-assessed physical activity [23]. Based on these results and the positive association between openness and discrepancy, individuals who scored higher in openness may overestimate their level of physical activity. However, older adults who scored higher in openness had better walking performance. Openness indicates willingness to try new things and individuals who score high in openness may engage in different types of physical activity [9]. The hip-worn accelerometer used in this study may not have captured all activities, such as strength training and cycling, and they were not worn during water-based activities. It is possible that this limitation of accelerometers explains the association between openness (and excitement seeking) and discrepancy.

Facets of conscientiousness were consistently related to physical activity and none of the facets stood out from the rest, supporting the previous studies about the positive association between conscientiousness and physical activity [7-9]. One explanation for this relationship is that individuals who are well-organized, dutiful, work hard to achieve their goals and have high self-discipline are more likely to implement their physical activity intentions [50]. Many facets of conscientiousness reflect self-control; hence, it is not surprising that conscientiousness has the opposite association with physical activity than impulsiveness. In contrast to impulsiveness, conscientiousness is linked to healthier weight $[49,51]$. People with low conscientiousness participate less in physical activity, which may lead to higher BMI and, in turn, to poorer walking performance and health outcomes [52]. In line with some previous studies [9], agreeableness was not associated with physical activity 


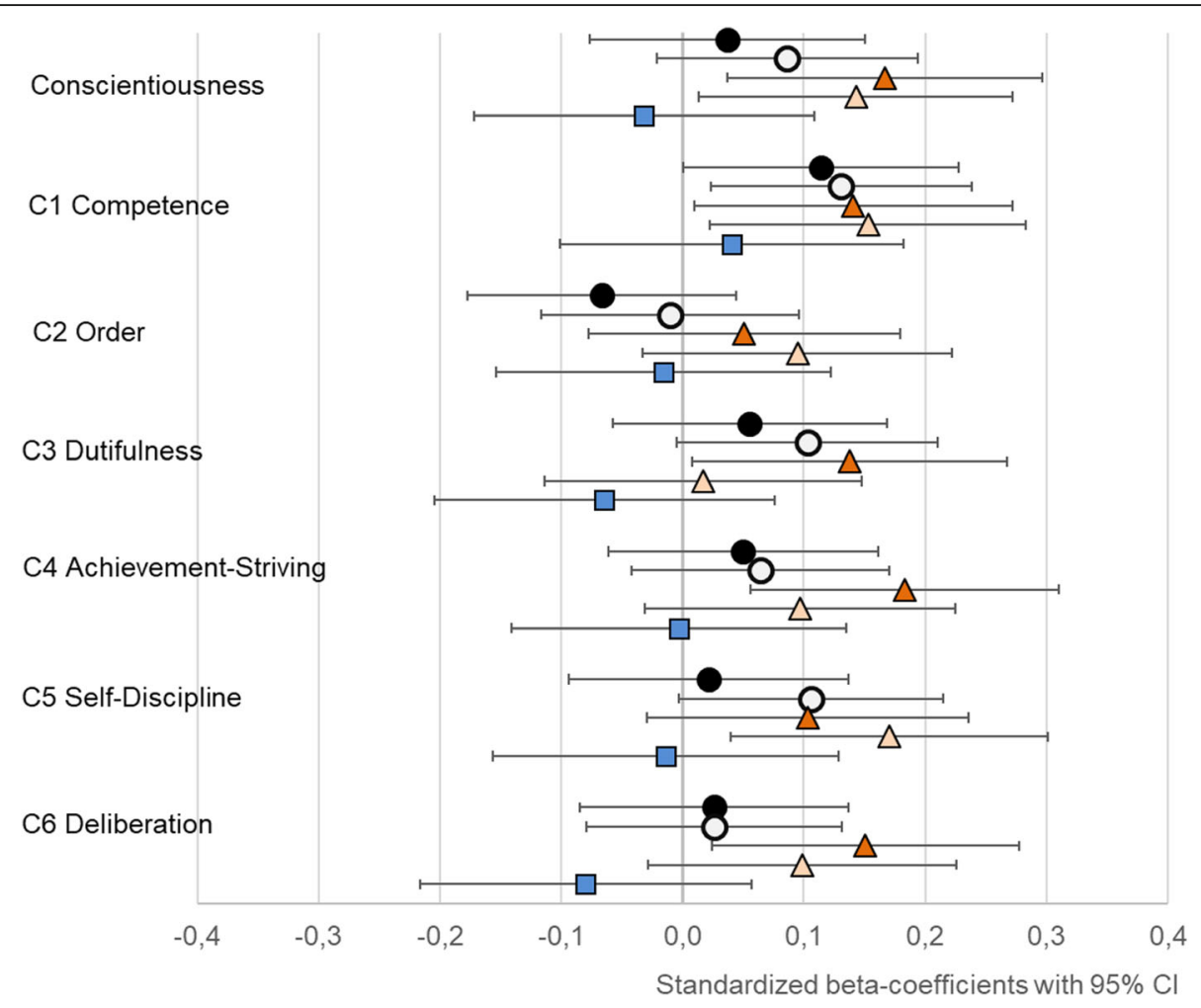

Walk $10 \mathrm{~m} \mathbf{O}$ Walk $6 \mathrm{~min} \Delta$ Light physical activity $\Delta$ Self-reported physical activity $\square$ Discrepancy

Fig. 5 The associations of conscientiousness with walking performance and physical activity (PA). Legend: Models adjusted by sex, age, education, BMI, diseases and intervention group, and for light PA also by moderate-to-vigorous physical activity

or walking performance. There was also no robust evidence of associations at the facet level.

This study has some limitations. While this was one of the largest studies to report on the associations between personality traits and accelerometer-measures, the sample size provided limited power to detect the typical small effects. In our relatively small sample, only the largest effects remained significant after accounting for multiple-testing, increasing the risk of false negative. Hence, these exploratory findings should be replicated with other samples and also with corresponding samples that have not participated in a training intervention. In addition, owing to the cross-sectional nature of this study, we cannot draw any conclusions about the causal relationships between the study variables.

Accelerometers have some limitations related to the cut-off points for older adults and their capability to capture different types of physical activities [30,31]. This might explain why we did not find an association with MVPA, which was surprising since the previous study on personality traits and accelerometer-assessed physical activity among older adults found associations only with MVPA and not with light physical activity [23]. Another explanation might be the low amount of MVPA among the participants of this study (mean $32.15 \mathrm{~min} / \mathrm{d} \pm$
21.01). For example, older adults in the study by Artese et al. [23] had almost four times more MVPA per day on average (mean $113.3 \pm 64.9$ ). However, our sample of older adults did not differ so much from the Finnish adult population (aged 20-69) who has about $45 \mathrm{~min}$ of MVPA per day on average [53].

Accelerometers record body acceleration in all daily activities (when a participant wears the accelerometer) [31] whereas self-reported MET-minutes used in this study asked about participation in leisure time exercise [44]. Therefore, it is possible that the discrepancy in part reflects participating in exercise but having very little amount of other daily activity. In any case, the discrepancy variable in this study was based on the difference between standardized variables and therefore indicated whether a participant reported the same amount of physical activity that was captured by accelerometers compared to the other participants.

Despite these limitations, this study was one of the first to investigate the role of personality in the discrepancy between physical activity measures and contributes to our understanding of how personality is linked to different measures of physical activity and walking performance. The major strengths of this study were the use of multiple measures of physical activity and walking 
performance and an in-depth assessment of personality at both trait- and facet-levels. Another relatively unique feature of this study was the use of a post-intervention sample. Unfortunately, we did not have access to the NEO-PI-3 at baseline data collection and therefore data collection occurred one-year after a physical activity intervention was used. As such, the findings illustrate the role of individual differences in personality for physical functioning even after older adults engaged in a standardized intervention to increase mobility.

\section{Conclusions}

This study has provided a deeper insight into how facetlevel personality tendencies explain part of the variability in physical activity and walking performance. Older adults with higher impulsiveness and lower self-control, who are less energetic and have less optimistic tendencies are at a higher risk for physical inactivity and walking limitations. Testing for personality characteristics in health care settings could help to identify individuals with higher risk for limitations. Interventions to promote physical functioning could also benefit from a more individually tailored approach. However, more research is needed on effective ways to promote physical functioning among people with personality characteristics that increase the risk for low physical functioning. In addition, a greater focus on personality characteristics could produce interesting findings that help us understand the discrepancy between self-reports and activity monitors.

\section{Supplementary Information}

The online version contains supplementary material available at https:/doi. org/10.1186/s11556-020-00251-9.

Additional file 1: Table S1. Associations of personality traits and facets with physical activity, discrepancy between physical activity measurements and walking performance. Description: Results of the regression analyses were five personality traits were tested in the same regression models.

Additional file 2: Table S2. Descriptive statistics for facets. Description: Descriptive statistics for facets.

Additional file 3: Table S3. Pearson bi-variate correlations between personality facets and physical functioning. Description: Pearson bi-variate correlations between personality facets and physical functioning.

Additional file 4: Table S4. Associations of personality traits and facets with accelerometer-assessed moderate-to-vigorous physical activity. Description: Results of the regression analyses for accelerometer-assessed moderate-to-vigorous physical activity.

\section{Abbreviations}

A1-A6: Facets of agreeableness; BMI: Body mass index; C1-C6: Facets of conscientiousness; E1-E6: Facets of extraversion; MET: Metabolic equivalent; MVPA: Moderate-to-vigorous physical activity; N1-N6: Facets of neuroticism; NEO-PI-3: NEO Personality Inventory - 3; 01-O6: Facets of openness; PASSWORD: Promoting safe walking among older people: the effects of a physical and cognitive training intervention vs. physical training alone on mobility and falls among older community-dwelling men and women

\section{Acknowledgements}

Not applicable.

\section{Authors' contributions}

KK and SS obtained funding for the study and contributed to the concept, design and data collection regarding the PASSWORD study. TK analyzed the data and drafted the manuscript. All authors were involved in the development of the research questions and interpretation of the results. All authors critically reviewed the manuscript, and read and approved the final version.

\section{Funding}

The preparation of the present article was funded by the Ministry of Education and Culture of Finland (KK: OKM/49/626/2017, OKM/72/626/2018, OKM/92/626/2019). The data collection of the PASSWORD study was funded by the Academy of Finland (SS: 296843) and by the Ministry of Education and Culture of Finland (KK: OKM/49/626/2017, OKM/72/626/2018). AT has received support from the National Institute on Aging of the National Institutes of Health (R21AG057917 and R01AG053297) and KK from the Academy of Finland (323541).

\section{Availability of data and materials}

Pseudonymized datasets are available on reasonable request. To request the data please contact Prof. Sarianna Sipilä (Sarianna.sipila@jyu.fi) (the PASS WORD data).

\section{Ethics approval and consent to participate}

The ethical committee of the Central Finland Hospital district provided an ethical statement about the PASSWORD on November 2016. Participants signed an informed consent before the assessments. Participants were allowed to withdraw their consent at any time during the study or for any individual part of the study.

\section{Consent for publication}

Not applicable.

\section{Competing interests}

The authors declare that they have no competing interests.

\section{Author details}

${ }^{1}$ Gerontology Research Center and Faculty of Sport and Health Sciences, University of Jyväskylä, Jyväskylä, Finland. ${ }^{2}$ Department of Geriatrics, College of Medicine, Florida State University, Tallahassee, FL, USA.

Received: 24 February 2020 Accepted: 4 November 2020

Published online: 24 November 2020

\section{References}

1. McPhee JS, French DP, Jackson D, Nazroo J, Pendleton N, Degens H. Physical activity in older age: perspectives for healthy ageing and frailty. Biogerontology. 2016;17:567-80.

2. Bennie JA, Pedisic Z, Suni JH, Tokola K, Husu P, Biddle SJ, et al. Self-reported health-enhancing physical activity recommendation adherence among 64,380 Finnish adults. Scand J Med Sci Sports. 2017;27:1842-53.

3. Dai $\mathrm{S}$, Carroll DD, Watson KB, Paul P, Carlson SA, Fulton JE. Participation in types of physical activities among US adults-National Health and nutrition examination survey 1999-2006. J Phys Act Health. 2015;12:S128-40.

4. Best JR, Liu-Ambrose T, Metti AL, Rosso AL, Satterfield S, Studenski S, et al. Longitudinal associations between walking speed and amount of selfreported time spent walking over a 9-year period in older women and men. J Gerontol Ser A Biol Sci Med Sci. 2018;73:1265-71.

5. McAdams DP. What do we know when we know a person? J Pers. 1995;63: 365-96.

6. McCrae RR, Costa PT. NEO inventories for the NEO personality Inventory-3, NEO five-factor Inventory-3, and NEP personality inventory-revised. Lutz: Psychological Assessment Resources; 2010.

7. Rhodes RE, Smith NEl. Personality correlates of physical activity: a review and meta-analysis. Br J Sports Med. 2006:40:958-65.

8. Sutin AR, Stephan Y, Luchetti M, Artese A, Oshio A, Terracciano A. The fivefactor model of personality and physical inactivity: a meta-analysis of 16 samples. J Res Pers. 2016;63:22-8. 
9. Wilson KE, Dishman RK. Personality and physical activity: a systematic review and meta-analysis. Pers Individ Diff. 2015;72:230-42.

10. Stephan Y, Sutin AR, Bovier-Lapierre G, Terracciano A. Personality and walking speed across adulthood: prospective evidence from five samples. Soc Psychol Personal Sci. 2018;9:773-80.

11. Tolea MI, Costa PT, Terracciano A, Ferrucci L, Faulkner K, Coday M, et al. Associations of openness and conscientiousness with walking speed decline: findings from the health, aging, and body composition study. J Gerontol Ser B Psychol Sci Soc Sci. 2012;67:705-11.

12. Tolea MI, Costa PT, Terracciano A, Griswold M, Simonsick EM, Najjar SS, et al. Sex-specific correlates of walking speed in a wide age-ranged population. J Gerontol Ser B Psychol Sci Soc Sci. 2010;65:174-84.

13. Terracciano A, Schrack JA, Sutin AR, Chan W, Simonsick EM, Ferrucci L. Personality, metabolic rate and aerobic capacity. PLoS One. 2013;8:e54746.

14. Löckenhoff CE, Terracciano A, Bienvenu OJ, Patriciu NS, Nestadt G, McCrae $\mathrm{RR}$, et al. Ethnicity, education, and the temporal stability of personality traits in the East Baltimore epidemiologic catchment area study. J Res Pers. 2008; 42:577-98.

15. Roberts BW, Mroczek D. Personality trait change in adulthood. Curr Dir Psychol Sci. 2008;17:31-5.

16. Rhodes RE, Pfaeffli LA. Personality and physical activity. The Oxford Handbook of Exercise Psychology. New York: Oxford University Press; 2012. p. 195-223.

17. Stephan Y, Boiché J, Canada B, Terracciano A. Association of personality with physical, social, and mental activities across the lifespan: findings from US and French samples. Br J Psychol. 2014;105:564-80.

18. Mõttus R, Sinick J, Terracciano A, Hřebíčková M, Kandler C, Ando J, et al. Personality characteristics below facets: a replication and meta-analysis of cross-rater agreement, rank-order stability, heritability, and utility of personality nuances. J Pers Soc Psychol. 2019;117:e35-50.

19. Paunonen SV, Ashton MC. Big five factors and facets and the prediction of behavior. J Pers Soc Psychol. 2001;81:524-39.

20. Paunonen SV, Haddock G, Forsterling F, Keinonen M. Broad versus narrow personality measures and the prediction of behaviour across cultures. Eur J Personal. 2003;17:413-33.

21. Watson D, Stasik SM, Ellickson-Larew S, Stanton K. Extraversion and psychopathology: a facet-level analysis. J Abnorm Psychol. 2015;124:432-46.

22. Adams TB, Mowen JC. Identifying the personality characteristics of healthy eaters and exercisers: a hierarchical model approach. Health Mark Q. 2005; 23:21-42.

23. Artese A, Ehley D, Sutin AR, Terracciano A. Personality and Actigraphymeasured physical activity in older adults. Psychol Aging. 2017;32:131-8.

24. Hoyt AL, Rhodes RE, Hausenblas HA, Giacobbi PR. Integrating five-factor model facet-level traits with the theory of planned behavior and exercise. Psychol Sport Exerc. 2009;10:565-72.

25. Rhodes RE, Courneya KS, Jones LW. Personality, the theory of planned behavior, and exercise: a unique role for extroversion's activity facet. J Appl Soc Psychol. 2002;32:1721-36.

26. Rhodes RE, Courneya KS, Jones LW. Personality and social cognitive influences on exercise behavior: adding the activity trait to the theory of planned behavior. Psychol Sport Exerc. 2004;5:243-54.

27. Rhodes RE, Courneya KS. Relationships between personality, an extended theory of planned behaviour model and exercise behaviour. $\mathrm{Br} \mathrm{J}$ Health Psychol. 2003;8:19-36.

28. Tolea MI, Terracciano A, Simonsick EM, Jeffrey Metter E, Costa PT, Ferrucci L. Associations between personality traits, physical activity level, and muscle strength. J Res Pers. 2012;46:264-70.

29. Rhodes RE, Courneya KS, Jones LW. The theory of planned behavior and lower-order personality traits: interaction effects in the exercise domain. Pers Individ Diff. 2005;38:251-65.

30. Schrack JA, Cooper R, Koster A, Shiroma E, Murabito JM, Rejeski WJ, et al. Assessing daily physical activity in older adults: unraveling the complexity of monitors, measures, and methods. J Gerontol A Biol Sci Med Sci. 2016;71:1039-48.

31. Strath SJ, Kaminsky LA, Ainsworth BE, Ekelund U, Freedson PS, Gary RA, et al. Guide to the assessment of physical activity: clinical and research applications. Circulation. 2013;128:2259-79.

32. Čukić I, Gale CR, Chastin SFM, Dall PM, Dontje ML, Skelton DA, et al. Crosssectional associations between personality traits and device-based measures of step count and sedentary behaviour in older age: the Lothian birth cohort 1936. BMC Geriatr. 2019;19:302.

33. Kekäläinen T, Laakkonen EK, Terracciano A, Savikangas T, Hyvärinen M, Tammelin TH, et al. Accelerometer-measured and self-reported physical activity in relation to extraversion and neuroticism: a cross-sectional analysis of two studies. BMC Geriatr. 2020;20:264.

34. Corbett DB, Valiani V, Knaggs JD, Manini TM. Evaluating walking intensity with hipworn accelerometers in elders. Med Sci Sports Exerc. 2016:48:2216-21.

35. Kowalski K, Rhodes RE, Naylor P-J, Tuokko H, MacDonald S. Direct and indirect measurement of physical activity in older adults: a systematic review of the literature. Int J Behav Nutr Phys Act. 2012;9:148.

36. Dyrstad SM, Hansen BH, Holme IM, Anderssen SA. Comparison of selfreported versus accelerometer-measured physical activity. Med Sci Sports Exerc. 2014;46:99-106

37. Watkinson C, van Sluijs EM, Sutton S, Hardeman W, Corder K, Griffin SJ. Overestimation of physical activity level is associated with lower BMl: a cross-sectional analysis. Int J Behav Nutr Phys Act. 2010;7:68.

38. Cerin E, Cain KL, Oyeyemi AL, Owen N, Conway TL, Cochrane T, et al. Correlates of agreement between accelerometry and self-reported physical activity. Med Sci Sports Exerc. 2016;48:1075-84.

39. Sipilä S, Tirkkonen A, Hänninen T, Laukkanen P, Alen M, Fielding RA, et al. Promoting safe walking among older people: the effects of a physical and cognitive training intervention vs. physical training alone on mobility and falls among older community-dwelling men and women (the PASSWORD study): design and methods of a randomized controlled trial. BMC Geriatr. 2018;18:215.

40. McCrae RR, Costa PTJ. NEO-PI-3 Persoonallisuusinventaari. Suomenkielisen version käsikirjaliite. [NEO-PI-3 Personality Inventory. The Finnish manual]. Hogrefe Psykologien Kustannus Oy; 2016.

41. Savikangas T, Tirkkonen A, Alen M, Rantanen T, Fielding RA, Rantalainen T, et al. Associations of physical activity in detailed intensity ranges with body composition and physical function a cross-sectional study among sedentary older adults. Eur Rev Aging Phys Act. 2020;17:4.

42. Vähä-Ypyä H, Vasankari T, Husu P, Suni J, Sievänen H. A universal, accurate intensity-based classification of different physical activities using raw data of accelerometer. Clin Physiol Funct Imaging. 2015;35:64-70.

43. Vähä-Ypyä H, Vasankari T, Husu P, Mänttäri A, Vuorimaa T, Suni J, et al. Validation of cut-points for evaluating the intensity of physical activity with accelerometry-based mean amplitude deviation (MAD). PLoS One. 2015;10: e0134813.

44. Kujala UM, Kaprio J, Sarna S, Koskenvuo M. Relationship of leisure-time physical activity and mortality: the Finnish twin cohort. JAMA. 1998;279:440-4.

45. Ainsworth B, Haskell W, Herrmann S, Meckes N, Bassett D, Tudor-Locke C, et al. 2011 compendium of physical activities: a second update of codes and MET values. Med Sci Sports Exerc. 2011;43:1575-81.

46. ATS Committee on Proficiency Standards for Clinical Pulmonary Function Laboratories. ATS Statement. Am J Respir Crit Care Med. 2002:166:111-7.

47. Benjamini Y, Hochberg Y. Controlling the false discovery rate: A practical and powerful approach to multiple testing. J R Stat Soc B. 1995;57:289-300.

48. Benjamini $Y$, Yekutieli $D$. The control of the false discovery rate in multiple testing under dependency. Ann Stat. 2001;29:1165-88.

49. Terracciano A, Sutin AR, McCrae RR, Deiana B, Ferrucci $L$, Schlessinger D, et al. Facets of personality linked to underweight and overweight. Psychosom Med. 2009;71:682-9.

50. Rhodes RE. The built-in environment: the role of personality and physical activity. Exerc Sport Sci Rev. 2006;34:83-8.

51. Sutin AR, Terracciano A. Five-factor model personality traits and the objective and subjective experience of body weight. J Pers. 2016;84:102-12.

52. Graham EK, Bastarache ED, Milad E, Turiano NA, Cotter KA, Mroczek DK. Physical activity mediates the association between personality and biomarkers of inflammation. SAGE Open Med. 2018;6:2050312118774990.

53. Husu P, Tokola K, Suni J, Vähä-Ypyä H, Mänttäri A, Vasankari T, et al. Suomalaisten objektiivisesti mitattu fyysinen aktiivisuus, paikallaanolo ja fyysinen kunto. [the objectively measured physical activity, sedentary behavior and physical fitness of Finns]. Helsinki: Ministry of Education and Culture, Finland; 2018

\section{Publisher's Note}

Springer Nature remains neutral with regard to jurisdictional claims in published maps and institutional affiliations. 